Check for updates

Cite this: RSC Adv., 2018, 8, 32132

Received 20th July 2018

Accepted 29th August 2018

DOI: 10.1039/c8ra06140h

rsc.li/rsc-advances

\section{A facile strategy for modifying boron nitride and enhancing its effect on the thermal conductivity of polypropylene/polystyrene blends $\uparrow$}

\author{
Xueliang Jiang, ${ }^{\text {ab }}$ Pengfei Ma, ${ }^{b}$ Feng You, iD *ab Chu Yao, iD ab Junlong Yao ${ }^{a b}$ \\ and Fangjun Liu ${ }^{\text {ab }}$
}

\begin{abstract}
Boron nitride (BN) possesses excellent thermal conductivity and remarkable insulating properties. However, poor compatibility between BN fillers and a polymer matrix and the weak ultimate mechanical properties of polymer composites are still big challenges to industrial applications in the thermal conductive field. In this paper, the dispersion of BN in a polystyrene (PS) matrix can be improved through the surface modification of $\mathrm{BN}$ by introducing in situ dispersion of polystyrene. Subsequently, the selective localization of modified BN in the PS phase can be realized. A co-continuous structure of polymer blends is designed to enhance the thermal conductivity of PS by introducing another polypropylene (PP) phase. The co-continuous PS/PP (60/ $40, w / w)$ phases can benefit further enhancement of thermal conductivity of PS due to the selective localization of modified BN in the PS phase. Furthermore, the thermal conductivity of PS/PP blends with only 14.5 wt\%-modified BN is 2 times higher than that of neat PP and $30 \%$ higher than that of PP/BN.
\end{abstract}

\section{Introduction}

Recently, polymer composites with unique physical and chemical properties have aroused much attention in electronic packaging, machine manufacturing and light-emitting diode (LED) applications. ${ }^{1-3}$ Thermal conductivity, insulation and flame resistance are inevitable and significant properties for related product performance. Unfortunately, many polymer materials are thermal insulating materials with average thermal conductivity lower than $0.3 \mathrm{~W} \mathrm{~m}^{-1} \mathrm{~K}^{-1}, 4,5$ which is discrepant of excellent heat dissipation. Therefore, much effort has been focused on conquering this drawback and to obtain high thermal conductive polymer materials, ${ }^{6,7}$ for instance, incorporating thermal conductive fillers.

The addition of conductive fillers to a polymer matrix is an efficient method to achieve high thermal conductive polymer materials. Generally, several fillers are often utilized such as aluminum nitride ${ }^{8}$ graphene, ${ }^{9}$ silicon carbide, ${ }^{10}$ carbon nanotubes (CNTs) ${ }^{11}$ boron nitride, ${ }^{12,13}$ and carbon black (CB). ${ }^{14}$ However, the thermal conductivity of polymer composites is still low when these conductive fillers are introduced. The main reasons for this phenomenon are ascribed to the weak interfacial interaction between filler and polymer matrix and the low

${ }^{a}$ Hubei Key Laboratory of Plasma Chemistry and New Materials, China

${ }^{b}$ School of Materials Science and Engineering, Wuhan Institute of Technology, Wuhan 430205, China.E-mail:490912761@qq.com; youfeng.mse@wit.edu.cn; chuyao@wit. edu.cn

† Electronic supplementary information (ESI) available. See DOI: 10.1039/c8ra06140h aspect ratio of fillers. ${ }^{15}$ Although high loading fillers can increase the thermal conductivity of polymer matrix, the deterioration in flexibility and high cost after increasing the content of thermal conductive filler restrain their potential applications. To date, several strategies have been proposed to enhance the thermal conductivity of polymer composites to control the filler loadings. For example, models of double percolation structure and 3D network structure have been employed..$^{16-18}$ Depending on the different methods, a 3D network can be constructed using the ice-templating method, ${ }^{19}$ core-shell segregated structure, $^{20} 3 \mathrm{D}^{- \text {foam }^{21}}$ and aerogels. ${ }^{22}$ Boron nitride (BN) is a new two-dimensional inorganic filler that has been incorporated into polymer matrices to fabricate thermal conductive polymer composites. A clear enhancing effect can be obtained due to its high thermal conductivity. ${ }^{23-28} \mathrm{Hu}^{29}$ et al. have fabricated three-dimensional BN network composites by combining controlled anisotropic freeze-casting with infiltration methods. In addition, inorganic filler coating polymer particle via electrostatic interaction $^{30}$ and $\pi-\pi$ self-assembling ${ }^{31}$ has also been used through hot-pressing in the temperature region of the glass-transition temperature and melting point to realize the conductive pathway. Alternatively, 3D foams of $\mathrm{BN}$ can be obtained by growth on $\mathrm{Ni}$ templates by using chemical vapor deposition, followed by etching of $\mathrm{Ni}^{21}$ This structure has been reported by Pettes, ${ }^{32}$ and it can decrease the internal contact thermal resistance. Therefore, constructing three-dimensional structures can effectively improve heat transfer and reduce the interfacial thermal resistance between fillers. Meanwhile, more heat transmission channels can be formed through the threedimensional structure. ${ }^{33}$ Moreover, two other methods that 
adopt characteristics found in nature have been employed to enhance the thermal conductivity of polymer matrix, and these are spider dragline silk and natural nacre. The first method reported by Wang demonstrated that thermal conductivity could increase with the enhancement of strain. ${ }^{34}$ This phenomenon occurs because of good orientation of crystalline regions and strong intrachain and interchain $\mathrm{H}$-bonds. The second way is to construct aligned brick-and-mortar structures, which results in outstanding mechanical strength and high Young's modulus for the final materials. Some attempts have been focused on generating high thermal conductive materials by imitating the structure of natural nacre. ${ }^{35}$ Moreover, designing a multilayered structure is another way to augment the filler content via controlling its interdiffusion in an immiscible polymer pair. ${ }^{36}$

Recently, a lot of research has been performed and previously reported on the preparation of thermal conductive polymer composites. $^{37-40}$ In our present study, a facile preparation of high thermal conductivity composite was reported by constructing a double percolation structure and controlling the distribution of modified BN in the polystyrene (PS) phase. First, PS microspherecoated BN was fabricated by in situ dispersion polymerization of silanized BN and styrene. Afterward, the modified BN and commercial PS were mixed through a two-roll mill and further processed with PP in a fixed proportion by melt blending. The resultant polymer composites exhibited higher thermal conductivity than PP/BN, where fillers can be constrained to disperse in the PS phase in the double percolation structure.

\section{Experimental}

\section{Materials}

h-BN platelets $\left(D_{50}=50 \mu \mathrm{m}\right)$ were purchased from Jonye Ceramic company, Zi Bo, China. 3-Aminopropyltriethoxysilane (KH550) was obtained from Sinopharm Chemical Regent Co. Ltd. Potassium persulfate and polyvinylpyrrolidone were purchased from Shanghai Lingfeng Chemical Reagent Co. Ltd. and Sinopharm Chemical Reagent Co. Ltd. Styrene was supplied by Sinopharm Chemical Regent Co. Ltd. to form the PS microsphere. Polypropylene (PP) and polystyrene (PS) spheres were provided by Sinopec Lanzhou Company and Sinopec Beijing Yanshan Company with melt flow indexes of $2.6 \mathrm{~g} / 10 \mathrm{~min}$ and $8.0 \mathrm{~g} / 10 \mathrm{~min}$, respectively.

\section{Synthesis of PS-coated BN}

The synthesis process of PS-coated BN was divided into two steps. In the first step, BN was modified by 3-aminopropyltriethoxysilane (KH550). To introduce hydroxyl on BN, BN flakes were thermally treated in a muffle furnace at $1000{ }^{\circ} \mathrm{C}$ for $2 \mathrm{~h}$ under an air atmosphere. ${ }^{41}$ Afterward, hydroxylated BN (10 $\mathrm{g}$ ), $1 \mathrm{ml} \mathrm{KH550}$ and $0.2 \mathrm{ml}$ formic acid (adjustment of the solution $\mathrm{pH}$ value) were put into a three-necked flask with an existing $100 \mathrm{ml}$ ethanol-water (9:1) mixed solution using ultrasonic treatment for $20 \mathrm{~min}$. The mixture solution was then stirred at $35{ }^{\circ} \mathrm{C}$ for $24 \mathrm{~h}$ to achieve silanization. Afterward, the resultant mixture was washed with ethanol, centrifuged and dried in oven at $50{ }^{\circ} \mathrm{C}$ for $8 \mathrm{~h}$. In the second step, $8 \mathrm{~g}$ purified styrene and $5.3 \mathrm{~g}$ KH550-modified $\mathrm{BN}$ (the $\mathrm{BN} / \mathrm{St}$ feeding ratio was 1 : 1.5) were poured into $100 \mathrm{ml}$ methyl alcohol; then, $0.15 \mathrm{~g}$ $\mathrm{K}_{2} \mathrm{~S}_{2} \mathrm{O}_{8}$ and $0.12 \mathrm{~g}$ PVP were added. The mixture was treated in an ultrasound cleaner for $30 \mathrm{~min}$. The in situ polymerization of styrene began at $70{ }^{\circ} \mathrm{C}$ for $6 \mathrm{~h}$. Finally, the resultant mixture was washed several times with distilled water and ethanol and dried in an oven at $50{ }^{\circ} \mathrm{C}$ for $8 \mathrm{~h}$. The synthesis procedure of PSmodified BN (BN@PS) is depicted in Scheme 1a.

\section{Preparation of BN@PS/PS binary composites, BN@PS/(PP/PS) ternary composites and $\mathrm{BN} /(\mathrm{PP} / \mathrm{PS})$ ternary composites}

The prepared BN@PS powder and PS pellets were melt blended to prepare BN@PS/PS binary composites by using a two-roll mixer at $130{ }^{\circ} \mathrm{C}$ for $30 \mathrm{~min}$. The weight fractions of BN@PS in BN@PS/PS binary composite were 0, $5 \mathrm{wt} \%, 10 \mathrm{wt} \%, 15 \mathrm{wt} \%$, $20 \mathrm{wt} \%, 30 \mathrm{wt} \%$ and $40 \mathrm{wt} \%$. Subsequently, BN@PS/(PP/PS) ternary composites were prepared by melt blending BN@PS/ PS binary composites with PP. The weight ratio of PP and PS (including the in situ polymerization PS granules) was fixed at $4: 6$ (Fig. S1 $\dagger$ ) and the actual content of BN in ternary composites can be calculated by using thermal gravimetric analysis. The detailed compositions are listed in Table 1, and the processing procedures were carried out in a Haake mixer at $175{ }^{\circ} \mathrm{C}$ and $50 \mathrm{rpm}$ for $10 \mathrm{~min}$. BN/(PP/PS) ternary composites with different contents of $\mathrm{BN}$ were also fabricated by using the same processing conditions for comparison. All composites were then compression molded at $180{ }^{\circ} \mathrm{C}$ under $15 \mathrm{MPa}$ to prepare testing samples. The schematic diagram of fabricating $\mathrm{BN} @ \mathrm{PS} /(\mathrm{PP} / \mathrm{PS})$ composites and $\mathrm{BN} /(\mathrm{PP} / \mathrm{PS})$ composites is presented in Scheme $1 \mathrm{~b}$ and $\mathrm{c}$ by contrast.

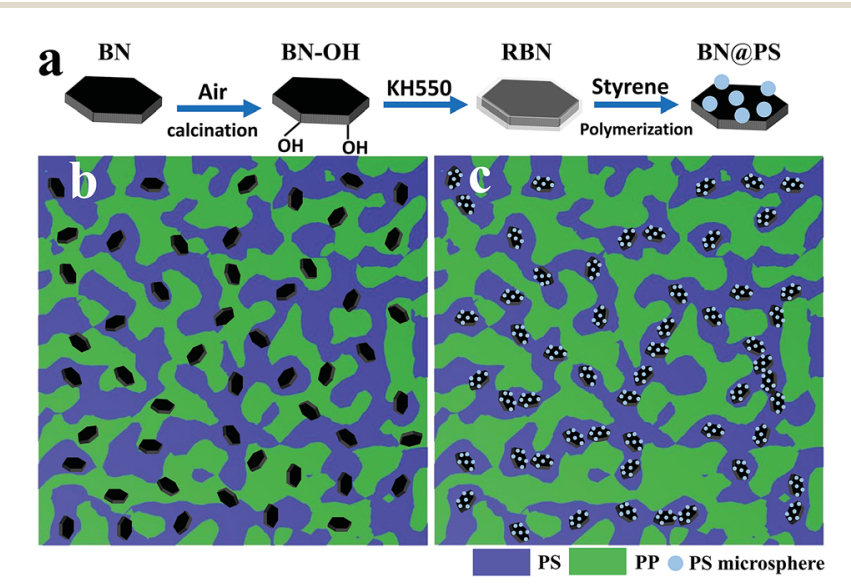

Scheme 1 (a) Schematic diagram of the modification of BN (b) and (c) Schematic illustration of the double percolated structure.

Table 1 The compositions of the sample

\begin{tabular}{lcll}
\hline & BN/wt\% & PS/wt\% & PP/wt\% \\
\hline BN@PS/(PP/PS) (3.37\%) & 3.37 & 58.0 & 38.63 \\
BN@PS/(PP/PS) (6.9\%) & 6.9 & 55.9 & 37.2 \\
BN@PS/(PP/PS) (10.6\%) & 10.6 & 53.6 & 35.8 \\
BN@PS/(PP/PS) (14.5\%) & 14.5 & 51.3 & 34.2
\end{tabular}




\section{Characterization}

BN flakes, RBN, and BN@PS were measured with IS50 FT-IR. Thermal gravimetric analysis (TGA) was carried out using a Netzsch STA 409 PC thermogravimetric analyzer under a nitrogen atmosphere. The temperature region was from room temperature to $800{ }^{\circ} \mathrm{C}$ with a heating rate of $10{ }^{\circ} \mathrm{C} \mathrm{min}^{-1}$. Morphology and microstructure of composites were observed by scanning electron microscopy (SEM, JSM-5510LV). The prepared test specimen was fractured in liquid nitrogen and then sputter-coated. To further analyze the elements in the samples and the relative amount of PS and BN in BN@PS sample, Energy Dispersive Spectrometry (EDS) was performed at an acceleration voltage of $15 \mathrm{eV}$. In addition, the fractured surfaces of BN@PS/(PP/PS) ternary composites were etched in chloroform solvent before SEM observation to remove the PS phase. Dynamic mechanical analysis (DMA) of composites was carried out by using a DMAQ800 (TA, American) instrument with a heating rate of $5{ }^{\circ} \mathrm{C} \mathrm{min}{ }^{-1}$ from $30{ }^{\circ} \mathrm{C}$ to $155^{\circ} \mathrm{C}$ at $1 \mathrm{~Hz}$. The thermal conductivity of the nanocomposites was measured on a DRL-III-C heat flow meter instrument (Xiangtan Xiangyi Instrument Co. Ltd) according to the standard ASTM D5470. ${ }^{42}$ Samples were prepared with a diameter of $30 \mathrm{~mm}$ and thickness of $2 \mathrm{~mm}$. Each sample was measured three times, and the average value was calculated.

\section{Results and discussion}

Fig. 1 shows the Fourier transform infrared (FTIR) spectra of BN, RBN, PS and BN@PS samples. Pristine BN exhibits two characteristic absorption peaks at $1369 \mathrm{~cm}^{-1}$ and $817 \mathrm{~cm}^{-1}$, which can be ascribed to the out of plane bending of $\mathrm{B}-\mathrm{N}-\mathrm{B}$ and the $\mathrm{B}-\mathrm{N}$ in-plane stretching vibration, respectively. After surface modification with KH550, RBN presents two absorption peaks at $985 \mathrm{~cm}^{-1}$ and $1068 \mathrm{~cm}^{-1}$ referred to $\mathrm{Si}-\mathrm{O}$ bond stretching due to the covalent combination between KH550 and BN. In addition, asymmetric and symmetric stretchings of $-\mathrm{CH}_{2}-$ at $2930 \mathrm{~cm}^{-1}$ and $2850 \mathrm{~cm}^{-1}$ can also be demonstrated by the modification of KH550 on BN surfaces. The small absorption

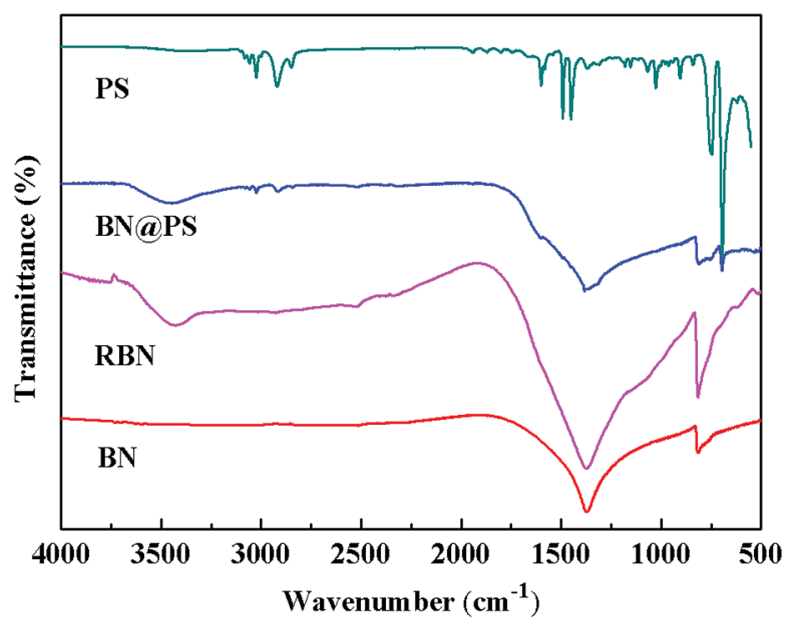

Fig. 1 FTIR of BN, RBN, PS and BN@PS. peak around $1600 \mathrm{~cm}^{-1}$ corresponds to the vibration of the benzene skeleton, indicating the generation of PS on $\mathrm{BN}$ surfaces for BN@PS.

The TGA curves of BN, RBN, BN@PS are shown in Fig. 2. It can be seen that $\mathrm{BN}$ displays almost no clear weight loss from room temperature to $800{ }^{\circ} \mathrm{C}$ because of its high thermal stability. However, a slight mass loss is exhibited for RBN at $100{ }^{\circ} \mathrm{C}$, indicating the decomposition of $\mathrm{KH} 550$ on the $\mathrm{BN}$ surface. The weight loss around $500{ }^{\circ} \mathrm{C}$ might be caused by the pyrolysis of chemically bonded silane molecules. As for BN@PS, two different degradations appear at $150-300{ }^{\circ} \mathrm{C}$ and $390-$ $470{ }^{\circ} \mathrm{C}$, respectively. One degradation results from the removal of weak hydroxyl groups and pyrolysis of chemically bonded silane molecules, and the other degradation is due to the thermal decomposition of PS. Approximately $45 \mathrm{wt} \%$ of mass loss can be observed through the TGA curve of BN@PS. Therefore, the content of BN in BN@PS is $55 \mathrm{wt} \%$ after calculating the experimental data. The actual content of BN in BN@PS/(PP/PS) ternary composites can also be calculated using this measurement.

BN@PS can be synthesized by incorporating modified BN during the polymerization of styrene monomer (Fig. 3a). From Fig. $3 \mathrm{~b}$ and $\mathrm{c}$, it can be seen that PS microspheres with a uniform particle size $(600-800 \mathrm{~nm})$ were prepared by dispersion polymerization of styrene. A uniform particle diameter distribution of PS microsphere was further demonstrated via SEM. Furthermore, good adhesion between PS microspheres with BN platelets can also be observed in Fig. 3c.

To confirm the structure of BN@PS, SEM combined with EDS mapping characterization was conducted for BN@PS powder. EDS mapping analysis was carried out on this region, which is shown as red rectangle in Fig. 4. The blue dots, red dots, and green dots corresponding to the distribution states of N, B and C indicated that the PS microspheres are successfully adsorbed on BN surface in accordance with the SEM results demonstrated in Fig. 3. The EDS analysis for the weight percentage of each element can be carried out. The results were 22.7 wt\%, 45.5 wt\%, 31.8 wt\% corresponding to B, C and $\mathrm{N}$. Therefore, the mass ratio of $\mathrm{BN}$ to PS was $1.2: 1$, which is well correlated with the above TGA results.

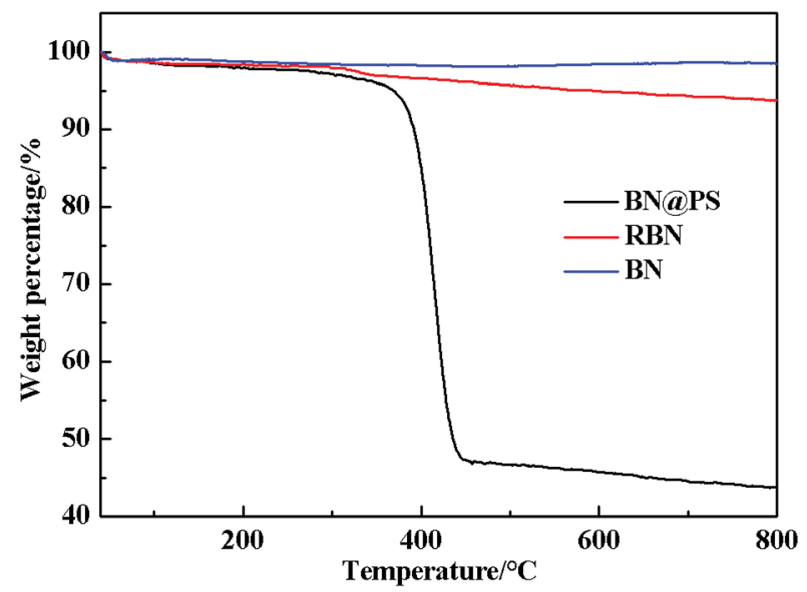

Fig. 2 TGA curves of BN, RBN and BNaPS. 

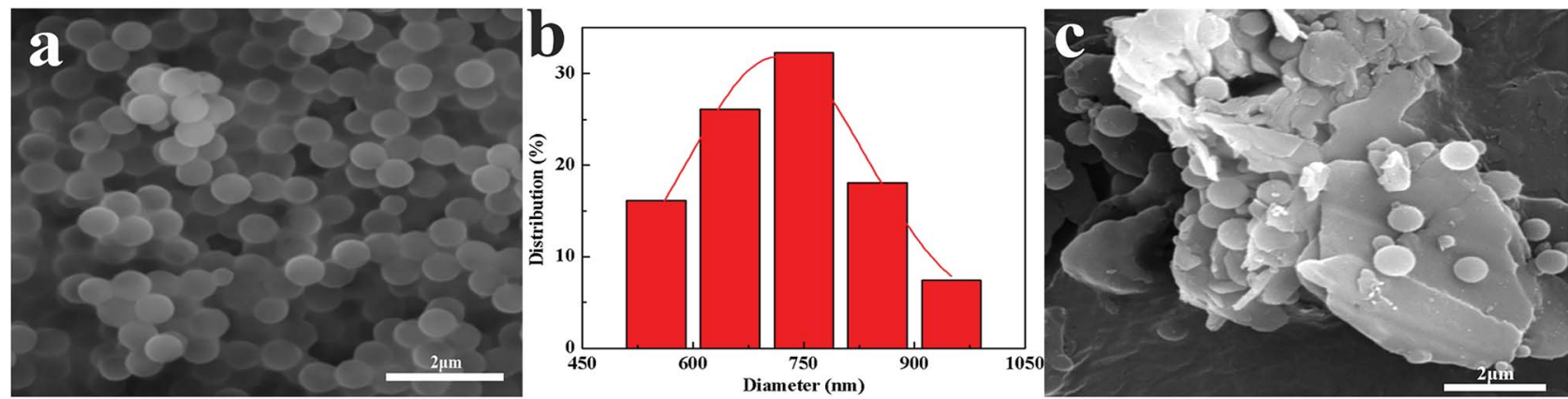

Fig. 3 SEM images of (a) PS (c) BNaPS and the distribution of the (b) PS diameter.
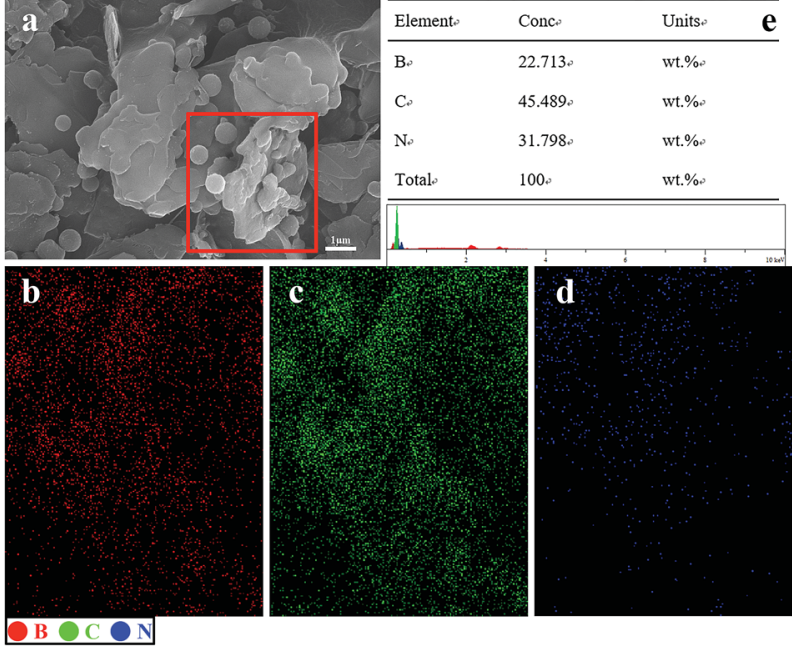

Fig. 4 SEM micrographs, element mapping and EDS analysis of BN@PS: (a) SEM, (b) B element, (c) C element, (d) N element, (e) EDS analysis.

The phase morphologies of PS/PP blends are presented in Fig. 5. The effect of different weight ratios of PS and PP on the phase morphology of PS/PP polymer blends was investigated in our laboratory (ESI, S1 $\dagger$ ). Finally, the weight ratio of PS/PP was fixed at $6: 4$ to achieve the co-continuous structure. It can be clearly seen that a co-continuous structure appeared for this weight ratio of polymer blends. In addition, BN@PS could be found to be selectively localized in the PS phase due to comparative large interfacial interaction between modified $\mathrm{BN}$ and PS (Fig. 5b and c). Consequently, the double percolation structure of thermal conductive network was generated, leading to the enhancement of thermal conductivity for PS. In other words, the incorporation of the PP component increased the actual loadings of BN in the PS phase.

Dynamic mechanical properties of BN@PS/(PP/PS) composites and $\mathrm{BN} /(\mathrm{PP} / \mathrm{PS})$ composites are given in Fig. 6 and 7. Fig. 6 shows the storage modulus of temperature with different $\mathrm{BN}$ contents. All curves exhibited the same tendency: the storage modulus increases with the increase in BN content. Owing to the high stiffness of the BN fillers, a large stress transferring effect from matrix to filler led to reinforcing effect on the final mechanical properties of composites. The storage modulus of $\mathrm{BN} @ \mathrm{PS} /(\mathrm{PP} / \mathrm{PS})$ composites was higher than that of $\mathrm{BN} /(\mathrm{PP} / \mathrm{PS})$ composites without modified $\mathrm{BN}$. The possible reason is that the interfacial interaction between modified BN and PS phase was higher than that of composites without modified BN. The glass transition temperature $\left(T_{\mathrm{g}}\right)$ of PS in different composites can also be found in Fig. 7. $T_{\mathrm{g}}$ of PP/PS blends was $125^{\circ} \mathrm{C}$; it increased with $\mathrm{BN}$ content for BN@PS/(PP/PS) composites. When the modified BN was $10.6 \mathrm{wt} \%, T_{\mathrm{g}}$ of BN@PS/(PP/PS) composites increased to $129^{\circ} \mathrm{C}$. By contrast, BN without treatment had negligible effect on $T_{\mathrm{g}}$ of $\mathrm{BN} /(\mathrm{PP} / \mathrm{PS})$ composites due to random dispersion of $\mathrm{BN}$ in the PS or PP phase. This difference in $T_{\mathrm{g}}$ further verified the selective localization of $\mathrm{BN}$ in the PS phase through in situ polymerization of the styrene monomer, which complied well with the above SEM observation. The selective localization of $\mathrm{BN}$ in the PS phase was good for the enhancement of $T_{\mathrm{g}}$ because of large interfacial interaction
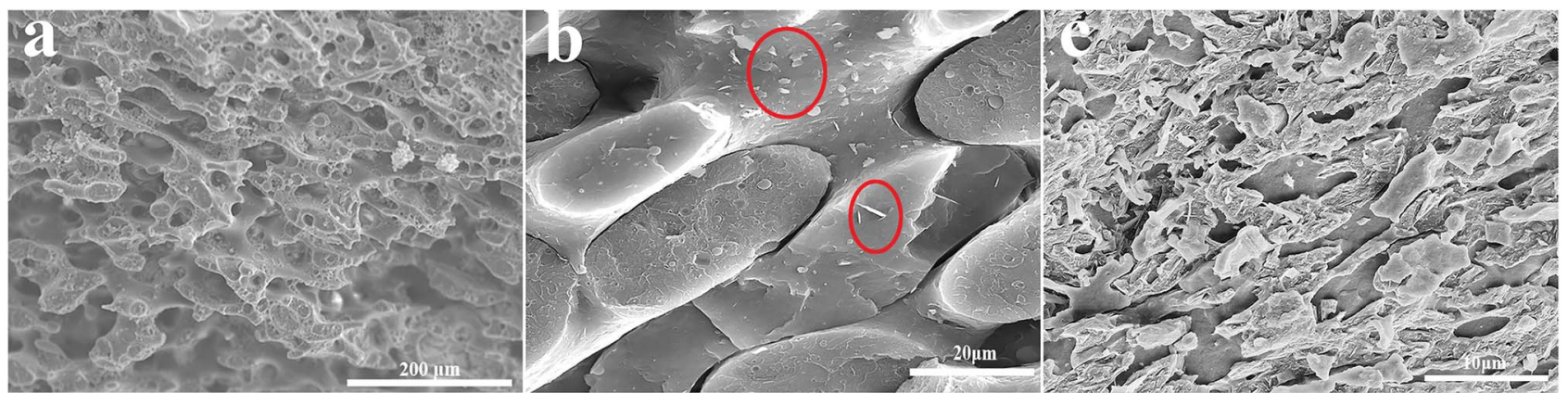

Fig. 5 SEM images of fractured surfaces for (a) PP/PS (4:6) and BN@PS/(PP/PS) composites with different contents of BN (b) 3.37 wt\%, (c) $14.5 \mathrm{wt} \%$. 

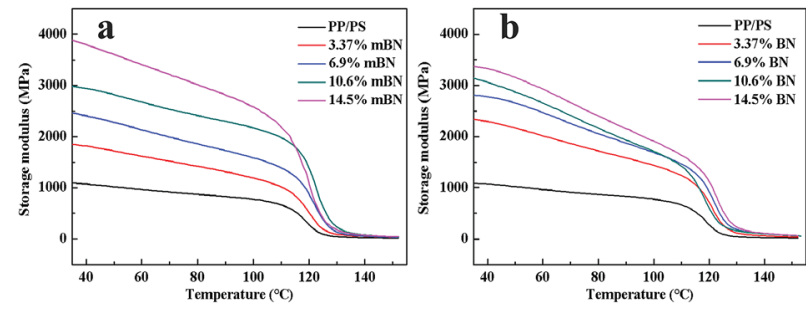

Fig. 6 Storage modulus versus temperature of (a) BNaPS/(PP/PS) composites and (b) BN/(PP/PS) composites.
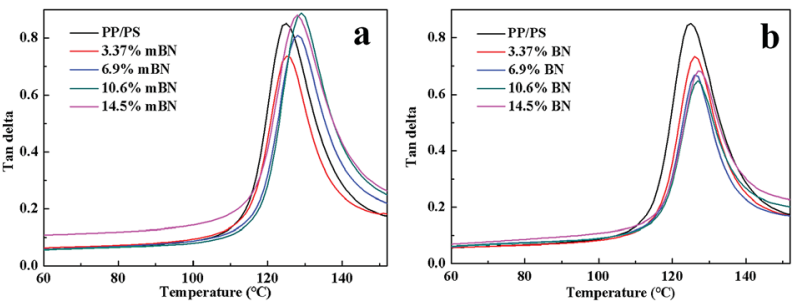

Fig. 7 Loss factors versus temperature of (a) BNaPS/(PP/PS) composites and (b) BN/(PP/PS) composites.

between the PS chain segment and modified $\mathrm{BN}$ as well as the large hindrance of chain segment motion.

Fig. 8 shows the thermal conductivity of $\mathrm{PP} / \mathrm{BN}, \mathrm{BN} /(\mathrm{PP} / \mathrm{PS})$ and BN@PS/(PP/PS) with different contents of BN. It can be seen that the thermal conductivity of these composites increased with BN content. Apparently, the thermal conductivity of $\mathrm{PP} / \mathrm{BN}$ phase binary composites was inferior to that of BN@PS/(PP/PS) or $\mathrm{BN} /(\mathrm{PP} / \mathrm{PS})$ ternary composites. Therefore, the double percolation structure of $\mathrm{BN}$ in $\mathrm{PS} / \mathrm{PP}$ with co-continuous structure is essential for enhancing the thermal conductivity of the composites by incorporating low content of thermal conductive filler. Moreover, the thermal conductivity of BN@PS/(PP/PS) composites was higher than that of $\mathrm{BN} /(\mathrm{PP} / \mathrm{PS})$ composites, indicating that the modification of BN could benefit the enhancement in thermal conductivity. The thermal conductivity of PS/PP blends with only $14.5 \mathrm{wt} \%$-modified BN was 2 times

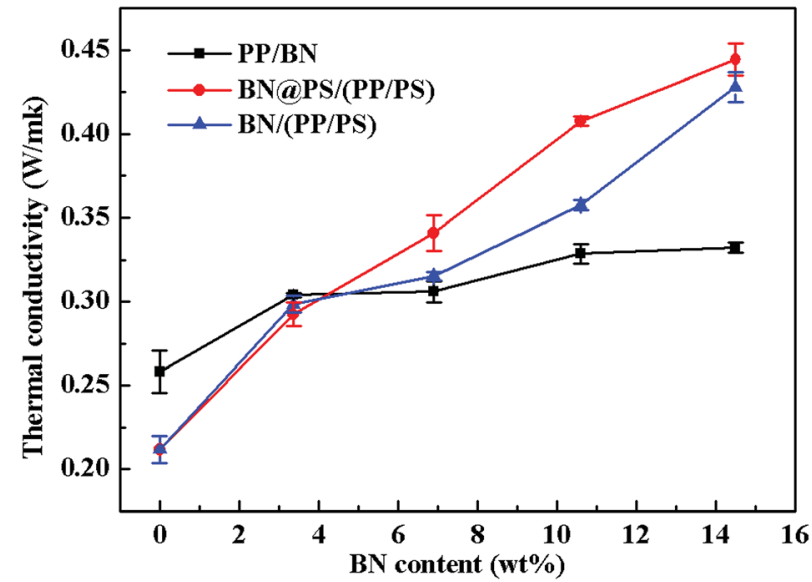

Fig. 8 Thermal conductivity of neat PP, PP/PS and composites with different contents of $\mathrm{BN}$. higher than that of neat PP and 30\% higher than that of PP/BN. The mechanism of the improvement in thermal conductivity of composites can be explained by the schematic description in Scheme 1. On one hand, modified BN enhanced the interfacial interaction between the fillers and PS matrix and improved the compatibility of fillers, leading to good dispersion of $\mathrm{BN}$ in polymer matrix. Simultaneously, the migration of BN to PP phase was inhibited due to large interfacial interaction between modified BN and PS. Therefore, the selective localization of BN resulted in the increase in weight loading for BN in the PS phase. Thus, the actual fraction of BN in the PS phase for BN@PS/(PP/ PS) composites was higher than that of $\mathrm{BN}$ without treatment in the PS phase for $\mathrm{BN} /(\mathrm{PP} / \mathrm{PS})$. On the other hand, the thermal interfacial resistance between fillers and matrix is the main reason for low thermal conductivity. Modified BN can reduce the interfacial resistance and enhance the heat dissipation efficiency. ${ }^{43}$ Therefore, this fabricating method is a facile way to enhance thermal conductivity through the low incorporation of modified BN. High thermal conductivity will be realized in our further study by combining two different dimensional thermal conductive fillers, which will include modified BN.

\section{Conclusions}

In this study, BN@PS/(PP/PS) ternary composites with high thermal conductivity were prepared through the doublepercolation structural design. The surface modification of BN was successfully conducted by polymerization of styrene on BN. The interfacial interaction between modified BN (PS-coated BN) and the PS phase was enhanced. A double-percolation structure of BN@PS/(PP/PS) ternary composites was obtained through cocontinuous PS/PP blends and selective localization of $\mathrm{BN}$ in the PS phase. SEM observation and DMA results showed that the selective localization of modified BN in the PS phase was realized. BN@PS/(PP/PS) ternary composites demonstrated higher thermal conductivity than $\mathrm{BN} /(\mathrm{PP} / \mathrm{PS})$ composites. The fabricating technique of high thermal conductive polymer composites by incorporating BN@PS and combining with a doublepercolation structure is a facile method to obtain high thermal conductivity materials with low filler loading.

\section{Conflicts of interest}

There are no conflicts to declare.

\section{Acknowledgements}

The financial support from the National Natural Science Foundation of China (No. 51273154), the Hubei Natural Science Foundation (No. 2017CFB289) and Graduate innovation fund of Wuhan Institute of Technology (No. CX2017006) is gratefully acknowledged.

\section{References}

1 S. Liu, B. Zhao, L. Jiang, Y. W. Zhu, X. Z. Fu, R. Sun, J. B. Xu and C. P. Wong, J. Mater. Chem. C, 2017, 6, 257-265. 
2 C. Pan, K. Kou, Q. Jia, Y. Zhang, G. Wu and T. Ji, Composites, Part B, 2017, 111, 83-90.

3 L. Ren, Q. Li, J. Lu, X. Zeng, R. Sun, J. Wu, J. B. Xu and C. P. Wong, Composites, Part A, 2018, 107, 561-569.

4 G. Wang, C. Wang, J. Zhao, G. Wang, C. B. Park and G. Zhao, Nanoscale, 2017, 9, 5996-6009.

5 Y. Yao, X. Zeng, R. Sun, J. B. Xu and C. P. Wong, ACS Appl. Mater. Interfaces, 2016, 8, 15645-15653.

6 Z.-G. Wang, F. Gong, W.-C. Yu, Y.-F. Huang, L. Zhu, J. Lei, J.-Z. Xu and Z.-M. Li, Compos. Sci. Technol., 2018, 162, 7-13.

7 C. Yu, J. Zhang, W. Tian, X. Fan and Y. Yao, RSC Adv., 2018, 8, 21948-21967.

8 Q. Wang, C. Bowen, W. Lei, H. Zhang, B. Xie, S. Qiu, M. Y. Li and S. Jiang, J. Mater. Chem. A, 2018, 6, 5040-5051.

9 S. Na, D. Jiao, S. Cui, X. Hou, D. Peng and L. Shi, ACS Appl. Mater. Interfaces, 2017, 9, 2924-2932.

10 D. Shen, Z. Zhan, Z. Liu, C. Yong, Z. Li, Y. Liu, D. Wen, K. Nishimura, C. Li and C. T. Lin, Sci. Rep., 2017, 7, 2606.

11 B. Yin, Y. Wen, H. Jia, J. Wang, Z. Xu and L. Ding, J. Polym. Eng., 2017, 37, 785-794.

12 M. Wang, Z. Jiao, Y. Chen, X. Hou, L. Fu, Y. Wu, S. Li, N. Jiang and J. Yu, Composites, Part A, 2018, 109, 321-329.

13 C. Yu, W. Gong, W. Tian, Q. Zhang, Y. Xu, Z. Lin, M. Hu, X. Fan and Y. Yao, Compos. Sci. Technol., 2018, 160, 199-207.

14 Y. J. Kim, Y. F. Tan and S. Kim, J. Compos. Mater., 2017, 52, 2047-2053.

15 T. M. L. Dang, C. Y. Kim, Y. Zhang, J. F. Yang, T. Masaki and D. H. Yoon, Composites, Part B, 2017, 114, 237-246.

16 M. Tanimoto, T. Yamagata, K. Miyata and S. Ando, ACS Appl. Mater. Interfaces, 2013, 5, 4374-4382.

17 S. Uchida, T. Murakami, T. Iwamura, R. Ishige and S. Ando, RSC Adv., 2017, 7, 15492-15499.

18 D.-L. Zhang, J.-W. Zha, C.-Q. Li, W.-K. Li, S.-J. Wang, Y. Wen and Z.-M. Dang, Compos. Sci. Technol., 2017, 144, 36-42.

19 X. Zeng, Y. Yao, Z. Gong, F. Wang, R. Sun, J. Xu and C. P. Wong, Small, 2015, 11, 6205-6213.

20 Y. Jiang, Y. Liu, P. Min and G. Sui, Compos. Sci. Technol., 2017, 144, 63-69.

21 M. Loeblein, S. H. Tsang, M. Pawlik, E. J. Phua, H. Yong, X. W. Zhang, C. L. Gan and E. H. Teo, ACS Nano, 2017, 11, 2033-2044.

22 H. Huang, P. Chen, X. Zhang, Y. Lu and W. Zhan, Small, 2013, 9, 1397-1404.
23 C. Yu, W. Gong, W. Tian, Q. Zhang, Y. Xu, Z. Lin, M. Hu, X. Fan and Y. Yao, Compos. Sci. Technol., 2018, 160, 199-207.

24 X. Yang, Y. Guo, X. Luo, N. Zheng, T. Ma, J. Tan, C. Li, Q. Zhang and J. Gu, Compos. Sci. Technol., 2018, 164, 59-64.

25 X. Yang, L. Tang, Y. Guo, C. Liang, Q. Zhang, K. Kou and J. Gu, Composites, Part A, 2017, 101, 237-242.

26 Y. Li, G. Xu, Y. Guo, T. Ma, X. Zhong, Q. Zhang and J. Gu, Composites, Part A, 2018, 107, 570-578.

27 J. Gu, C. Liang, J. Dang, W. Dong and Q. Zhang, RSC Adv., 2016, 6, 35809-35814.

28 J. Gu, S. Xu, Q. Zhuang, Y. Tang and J. Kong, IEEE Trans. Dielectr. Electr. Insul., 2017, 24, 784-790.

29 J. Hu, Y. Huang, Y. Yao, G. Pan, J. Sun, X. Zeng, R. Sun, J. B. Xu, B. Song and C. P. Wong, ACS Appl. Mater. Interfaces, 2017, 9, 13544-13553.

30 X. Wang and P. Wu, ACS Appl. Mater. Interfaces, 2017, 9, 19934-19944.

31 K. Wu, Y. Li, R. Huang, S. Chai, F. Chen and Q. Fu, Compos. Sci. Technol., 2017, 151, 193-201.

32 M. T. Pettes, H. Ji, R. S. Ruoff and L. Shi, Nano Lett., 2012, 12, 2959-2964.

33 X. Li, L. Shao, N. Song, L. Shi and P. Ding, Composites, Part A, 2016, 88, 305-314.

34 X. Huang, G. Liu and X. Wang, Adv. Mater., 2012, 24, 14821486.

35 G. Pan, Y. Yao, X. Zeng, J. Sun, J. Hu, R. Sun, J. B. Xu and C. P. Wong, ACS Appl. Mater. Interfaces, 2017, 9, 3300133010.

36 S.-Y. Yang, Y.-F. Huang, J. Lei, L. Zhu and Z.-M. Li, Composites, Part A, 2018, 107, 135-143.

37 J. Huang, Y. Zhu, L. Xu, J. Chen, W. Jiang and X. Nie, Compos. Sci. Technol., 2016, 129, 160-165.

38 N. Song, D. Jiao, P. Ding, S. Cui, S. Tang and L. Y. Shi, J. Mater. Chem. C, 2015, 4, 305-314.

39 W. Zhou, Y. Gong, L. Tu, L. Xu, W. Zhao, J. Cai, Y. Zhang and A. Zhou, J. Alloys Compd., 2017, 693, 1-8.

40 J. Gu, N. Li, L. Tian, Z. Lv and Q. Zhang, RSC Adv., 2015, 5, 36334-36339.

41 Z. Cui, A. J. Oyer, A. J. Glover, H. C. Schniepp and D. H. Adamson, Small, 2014, 10, 2352-2355.

42 X. Yang, C. Liang, T. Ma, Y. Guo, J. Kong, J. Gu, M. Chen and J. Zhu, Adv. Compos. Hybrid Mater., 2018, 1, 207-230.

43 Y. Jiang, X. Shi, Y. Feng, S. Li, X. Zhou and X. Xie, Composites, Part A, 2018, 107, 657-664. 